\title{
Probing Nanoparticle/Membrane Interactions by Combining Amphiphilic Diblock Copolymer Assembly and Plasmonics
}

\author{
Amelie H.R. Koch, Svenja Morsbach, Tristan Bereau, Gaëtan Lévêque, Hans-Jürgen Butt, \\ Markus Deserno, Katharina Landfester, and George Fytas*
}

Cite This: J. Phys. Chem. B 2020, 124, 742-750

Read Online

ABSTRACT: Understanding the interactions between nanoparticles (NPs) and boundaries of cells is crucial both for their toxicity and therapeutic applications. Besides specific receptor-mediated endocytosis of surface-functionalized NPs, passive internalization is prompted by relatively unspecific parameters, such as particle size and charge. Based on theoretical treatments, adhesion to and bending of the cell membrane can induce NP wrapping. Experimentally, powerful tools are needed to selectively probe possible membrane-NP motifs at very dilute conditions and avoid dye labeling. In this work, we employ surface resonance-enhanced

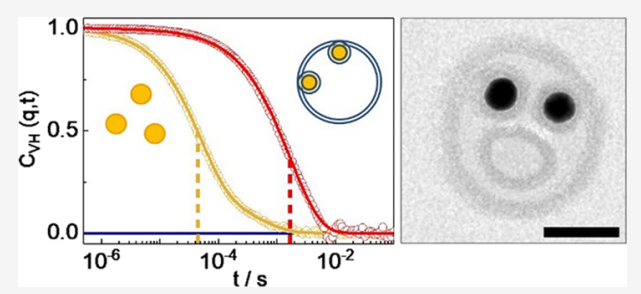
dynamic light scattering, surface plasmon resonance, electron microscopy, and simulations for sensing interactions between plasmonic AuNPs and polymersomes. We distinguish three different interaction scenarios at nanomolar concentrations by tuning the surface charge of AuNPs and rationalize these events by balancing vesicle bending and electrostatic/van der Waals AuNP and vesicle adhesion. The clarification of the physical conditions under which nanoparticles passively translocate across membranes can aid in the rational design of drugs that cannot exploit specific modes of cellular uptake and also elucidates physical properties that render nanoparticles in the environment particularly toxic.

\section{INTRODUCTION}

The growing exposure of humans (and other living organisms) to an ever-growing spectrum of artificially produced nanoparticles (NPs) has sparked concerns about their toxicity, ${ }^{1}$ which is often related to an NP's ability to enter cells and interfere with normal processes once inside. This is, to some extent, the flip side of numerous applications where one expressly wishes to guide certain NPs into cells or tissues, for instance, when these NPs carry drugs ${ }^{2}$ or are used for medical imaging and diagnostics. ${ }^{3,4}$ Understanding how NPs interact with lipid membranes, the boundaries of all living cells, is hence crucial both for beneficial applications and to mitigate or avoid potential deleterious side effects. While both in vivo ${ }^{5}$ and in vitro ${ }^{6}$ studies have been performed for a wide range of different NPs, the mechanisms of entry and subsequent intracellular trafficking are still not very well understood. ${ }^{7,8}$

Most cells can actively take up NPs from outside via receptor-mediated endocytosis. ${ }^{9,10}$ In this active process, a complex cellular machinery is triggered to actively engulf and internalize an object once certain ligands on its surface bind to specific receptors on a cell's plasma membrane. But many NPs do not have specific ligands, and uptake is prompted by relatively unspecific cues (such as particle size, charge, and surface chemistry) that remain a source of debate. ${ }^{11-13}$ However, cells can also passively ingest particles that adhere strong enough to overcome the elastic penalty for membrane bending. This type of adhesion-induced particle wrapping has been widely studied within continuum elastic treatments (using both analytical and numerical techniques), looking, for instance, at simple spherical particles ${ }^{14-16}$ or particles covered with discrete binding sites ${ }^{17-19}$ or more complicated geometric or elastic properties. ${ }^{20-23}$ The problem has also been treated in many coarse-grained simulation studies, ${ }^{24-31}$ which strive to elucidate aspects that are difficult to capture analytically, such as membrane fluctuations, particle cooperativity, and bilayer disruption.

A recurring theme in all this work is that a particle can end up in either one of three distinct states: unbound, partially wrapped, or fully enveloped, as schematically shown in Figure 1. This outcome is mostly determined by physical properties of the system (such as adhesion strength, particle geometry, membrane elasticity, spontaneous curvature, and tension). The ultimate fate of the fully wrapped state is less clear because actual internalization requires membrane fission. This topology-changing process is challenging to capture in the continuum theory, but it has been studied by treating the two individual membrane leaflets separately and working out the complex energetics of nonbilayer intermediates (such as stalks), ${ }^{32,33}$ for which lipid tilt turned out to be essential. ${ }^{34}$ Very recent experiments have shown that the energy barrier of

Received: November 7, 2019

Revised: January 10, 2020

Published: January 17, 2020 

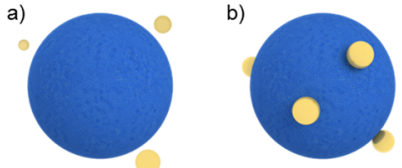

c)

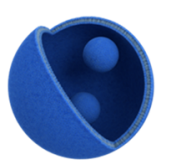

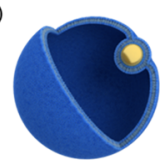

Figure 1. (a-d) Schematic representations of possible interaction scenarios. From left to right: no interaction between vesicles and NPs; decoration of vesicle with NPs; uptake and detachment of the endosome from the inner side of the membrane; and uptake but endosome stays attached to the inner side of the membrane.

spontaneous lipid bilayer fusion is on the order ${ }^{35}$ of $30 k_{\mathrm{B}} T$, too large to occur on its own, but well within the range of the characteristic energies that can be liberated by simple cellular fusion and fission machineries, which explains why in nature these processes are always catalyzed by specialized proteins. ${ }^{9}$

Owing to its biomedical relevance, the impact of NPs on biomembranes is usually studied on live cells, but this makes it difficult to disentangle passive entry from active cellorchestrated uptake. On the one hand, nanoparticles can enter red blood cells ${ }^{36}$ and macrophages treated with cytochalasin D (a potent inhibitor of actin polymerization and hence endocytosis), ${ }^{37}$ which illustrates the possibility of passive entry processes, since both systems lack an active endocytic machinery. On the other hand, it has also been shown that passive uptake is not inevitable: gold nanoparticles (with a diameter of up to $15 \mathrm{~nm}$ ) did not spontaneously enter liposomes formed from biomimetic model membranes, ${ }^{38}$ while larger hydrophilic silica particles (with a diameter between 30 and $200 \mathrm{~nm}$ ) can fully enter DOPC model membrane vesicles. ${ }^{39}$ In addition, the interplay between global constraints on vesicle volume and the extent of nanoparticle binding ${ }^{40}$ or the cooperative interaction between multiple nanoparticles bound to the membrane $e^{40,41}$ adds a significant level of complexity.

To further elucidate the mechanistic details of the entry process, especially the transition between full wrapping and complete internalization, we must better understand the local morphology a membrane takes when it envelops a particle, a challenging nanoscale imaging problem. However, considering that the essential physics is governed by generic aspects (particle geometry, membrane elasticity, and adhesion energy), a conceivable path forward is to study systems that exhibit the same behavior but at a larger scale: membranes that are selfassembled from amphiphilic block copolymers, ${ }^{42}$ which tend to be thicker than ordinary lipid membranes. Since (at a fixed value of the Young modulus) the bending rigidity of such fluid elastic surfaces scales with the cube of their thickness, ${ }^{43}$ while the characteristic nanoparticle size of adhesion and wrapping both increase with the square root of the rigidity, ${ }^{15,16}$ we see that thicker membranes necessarily shift the entire wrapping scenario to larger scales, from both a geometric and an elastic point of view.

Similar to lipids, amphiphilic block copolymers self-organize in water into so-called polymersomes, whose main advantage over liposomes is long-term stability. ${ }^{44}$ Their chemical, physical, and mechanical properties can be synthetically tuned to match the requirements of the desired membrane. Uptake of silica and polystyrene nanospheres into polymersomes formed from poly(dimethylsiloxane)-block-poly(2-methyl-oxazoline) (PDMS- $b$-PMOXA) has been demonstrated. ${ }^{45,46}$ By choosing a suitable pair of block copolymer membranes and nanoparticles, many generic aspects of the wrapping problem can be studied in exquisite detail.

In contrast to previous work, ${ }^{39,45-47}$ we, here, use plasmonic metal gold nanoparticles (AuNPs) in this study to devise an exceptionally sensitive system for studying vesicle/nanoparticle interactions. Among metal particles, gold colloids are probably the most prominent examples, and they have been used in clinical therapy as early as 1920s. AuNPs can be synthesized in many different shapes, including spheres and rods, but also more advanced geometries. ${ }^{48-51}$ AuNPs exhibit a number of physical properties, which make them very suitable for medical applications, such as the absorbance of light in the visible and near-infrared regions, ${ }^{52,53}$ the high optical scattering intensities, $^{54}$ the large sensitivity to refractive index changes for biosensing, ${ }^{55}$ absorbance of X-rays, ${ }^{56}$ and the ability to transform absorbed light into heat. ${ }^{57,58}$ In addition, the surface of AuNPs can be modified easily due to thiol chemistry. Here, the latter and the plasmonic properties are exploited to gain detailed insights into the fate of differently modified spherical AuNPs upon vesicle interaction (see Figure 1). We do not consider insertion of NP clusters into vesicle bilayers ${ }^{59}$ and supported lipid bilayer formation ${ }^{60}$ since the NP diameter was either too large or too small for these events. Moreover, we could not detect both NPs and vesicles separately, so we cannot report information about the fate of the endosome.

In this work, we employ surface resonance-enhanced dynamic light scattering (DLS), a powerful and highly sensitive technique for sensing interactions between plasmonic AuNPs and polymersomes, making it possible to distinguish multiple interaction scenarios from a single experiment at nanomolar concentrations. Additionally, information on the geometry of the nanoparticle/vesicle complex can be obtained from the same experiment by computer-assisted analysis of the scattering patterns. Vesicles and nanoparticles can be distinguished without dye labeling as it would be needed in fluorescence-based techniques. The findings from DLS are confirmed by cryo-TEM imaging and UV-vis measurements. The introduced system offers a basis for experimental proof of many theoretical studies.

\section{METHODS}

Synthesis of the Amphiphilic Block Copolymer and the Spherical Gold Nanoparticles. Poly(dimethylsiloxane)block-poly(2-methyl-oxazoline) (PDMS-b-PMOXA) was synthesized according to Egli et al. by sequential ring-opening polymerization. ${ }^{61}$ The polymerization reaction was terminated by adding piperazine and hence resulting in a piperazylfunctionalized end group. The number-average degrees of polymerization were 65 for PDMS and 17 for PMOXA, amounting to a molecular weight of $M_{\mathrm{W}} \approx 6600 \mathrm{~g} \mathrm{~mol}^{-1}$. Polymersomes were prepared using the film rehydration method $^{62}$ followed by extrusion through polycarbonate membranes with defined pore sizes, as described by Jaskiewicz et al. $^{46} \mathrm{~A}$ stock of gold spheres was synthesized according to the literature procedure. ${ }^{63} \mathrm{SH}-\mathrm{PEG}-\mathrm{OCH}_{3}\left(M_{\mathrm{W}}=5079 \mathrm{Da}\right)$ and cetyltrimethylammonium bromide $(\mathrm{CTAB})$ were bound to the particles by overnight incubation at room temperature as described by Hanauer et al. ${ }^{64}$ Complete description and characterization of the AuNPs are given in the literature. ${ }^{65}$

Dynamic Light Scattering (DLS). The normalized light scattering intensity $(I(q, t))$ autocorrelation function $G(q, t) \equiv$ $\langle I(q, t) I(q)\rangle /\left.I I(q)\right|^{2}$ was recorded over a broad time range $\left(10^{-7}-10^{3} \mathrm{~s}\right)$ at different scattering wave vectors $q$ with an 
Table 1. Characteristic Dimensions of the Vesicles and the Au Nanospheres with CTAB, Citrate, and Grafted PEG Ligands, their $\zeta$ Potential, and the Wavelength at the Absorption Peak of the Surface Plasmon Resonance

\begin{tabular}{|c|c|c|c|c|c|c|}
\hline sample & $c(\mathrm{nM})$ & $R_{\mathrm{h}}(\mathrm{nm})^{a}$ & $R_{\mathrm{VH}} / R_{\mathrm{VV}}$ & $R_{\mathrm{r}}(\mathrm{nm})$ & $\zeta$ potential $(\mathrm{mV})$ & $\lambda_{\max }(\mathrm{nm})$ \\
\hline vesicles & 0.045 & $135 \pm 3$ & & & $+27.5 \pm 1.1$ & \\
\hline $\mathrm{Au}-\mathrm{CTAB}$ & 0.2 & $23 \pm 0.7$ & 0.06 & $32.7 \pm 0.3$ & $+53.9 \pm 8.1$ & 524 \\
\hline $\mathrm{Au}-\mathrm{PEG}_{107}$ & 0.2 & $37 \pm 1.4$ & 0.02 & $40.0 \pm 0.6$ & $-2.1 \pm 3.9$ & 526 \\
\hline Au-citrate & 0.2 & $24 \pm 0.5$ & 0.08 & $34.8 \pm 0.7$ & $-29.2 \pm 4.7$ & 524 \\
\hline
\end{tabular}

${ }^{a}$ The negligible anisotropic scattering of the spherical vesicles precludes access to $R_{\mathrm{r}}$.

$\mathrm{ALV} / \mathrm{LSE}-5004$ goniometer/correlator setup using a $\mathrm{HeNe}$ laser with wavelength $\lambda=632 \mathrm{~nm}$. The scattering vector $q=k_{\mathrm{s}}$ $-k_{\mathrm{i}}$ with $k_{\mathrm{s}}$ and $k_{\mathrm{i}}$ being the wave vectors of the scattered and incident light, respectively, has a magnitude of $q=(4 \pi n)$ $\lambda) \sin (\theta / 2)$ ( $n$ and $\theta$ are the solution refractive index and the scattering angle, respectively). We have performed both polarized (VV) and depolarized (VH) photon correlation spectroscopy (PCS) experiments using a vertically (V) polarized incident laser beam and selected the scattered light polarized vertically (VV configuration) and horizontally (VH configuration) to the scattered plane $\left(k_{\mathrm{i}}, k_{\mathrm{f}}\right)$. The measurements were carried out at temperature $T=20^{\circ} \mathrm{C}$. For spherical NPs, the translational diffusion coefficient $D^{\mathrm{t}}$ is directly obtained from the diffusive relaxation rate $\Gamma_{\mathrm{VV}}=D^{\mathrm{t}} q^{2}$ of the isotropic relaxation function. For this case, no scattering in $\mathrm{VH}$ can be observed. For anisotropic particles, rotational motion is unequal to the translational motion and can be observed in $\mathrm{VH}$ geometry. $D^{\mathrm{r}}$ is the rotational diffusion coefficient and $\Gamma_{\mathrm{VH}}$ $=6 D^{r}+D^{t} q^{2}$. The isotropic and anisotropic relaxation functions are

$$
\begin{aligned}
& C_{\mathrm{VH}}(q, t)=\exp \left[-\Gamma_{\mathrm{f}} t\right] \\
& C_{\mathrm{VV}}(q, t)=a_{\mathrm{f}} \exp \left[-\Gamma_{\mathrm{f}} t\right]+a_{\mathrm{s}} \exp \left[-\Gamma_{\mathrm{s}} t\right]
\end{aligned}
$$

where $\Gamma_{\mathrm{f}}=\Gamma_{\mathrm{VH}}$ and $\Gamma_{\mathrm{s}}=D^{\mathrm{t}} q^{2}$ are the relaxation rates for the fast (f) and slow (s) processes, respectively. The VV scattering includes both isotropic and anisotropic contributions, and therefore, $C_{\mathrm{VV}}$ becomes bimodal, with fast and slow processes characterized by amplitudes $a_{\mathrm{f}}$ and $a_{\mathrm{s}}=\left(1-a_{\mathrm{f}}\right)$ and rates $\Gamma_{\mathrm{f}}$ and $\Gamma_{s}$.

The dilution of vesicles displays a purely diffusive behavior with a relaxation rate $\Gamma=D^{t} q^{2}$ (Figure $S 1 b$ ), whereas the representation of the polarized scattering intensity $R_{\mathrm{VV}}(q)$ (Figure S1a) by the form factor of a vesicle leads to the estimation of the molecular weight and the radius $R$. The Holtzer plot in the inset of Figure S1a justifies that the whole vesicle is probed at low scattering $q$ values. For the vesicle/AuCTAB solution, the $R_{\mathrm{VV}}(q)$ pattern is virtually represented by the sum of the mixture constituents, vesicle and $\mathrm{Au}-\mathrm{CTAB}$ solutions, as shown in Figure S2a. Consistently, the dynamics (Figure S2b) are attributed to the noninteracting Au-CTAB NPs and vesicles.

Computational Scattering Profiles for Gold/Polymersome Systems. Vesicles and nanoparticles were assigned a scattering length density $\rho$, while the environment was set at $\rho$ $=0$, thereby effectively working with contrasts and greatly reducing the computational cost. We modeled vesicles as shells of radii $R_{\text {ves }}{ }^{\text {inner }}$ and $R_{\text {ves }}{ }^{\text {outer }}$, where the scattering length density of the inner sphere was set to the environment (i.e., $\rho=0$ ), while nanoparticles were described as spheres of size $R_{\mathrm{NP}}$. We discretized vesicles and nanoparticles on a cubic grid of volume $L^{3}=600 \mathrm{~nm}^{3}$ with grid spacing $a=18 \mathrm{~nm}$ (i.e., a small enough length scale to probe details up to the maximum wave vector considered), assigning the scattering length density on each grid point depending on any object present. We optimized the radius of the vesicle to best reproduce the curvature of its experimental scattering profile, although its thickness was set to $R_{\text {ves }}{ }^{\text {outer }}-R_{\text {ves }}^{\text {inner }}=20 \mathrm{~nm}$, while we enforced the radius of nanoparticles to $R_{\mathrm{NP}}=20 \mathrm{~nm}$. The scattering form factor was determined using the Debye formula

$$
P(q)=\frac{1}{z^{2}} \sum_{i=1}^{z} \sum_{j=1}^{z} \rho_{i} \rho_{j} \frac{\sin \left(q r_{i j}\right)}{q r_{i j}}
$$

where $z$ is the number of particles, and $r_{i j}$ is the distance between particles $i$ and $j$. We studied both decoration and uptake mechanisms of nanoparticles on the polymersome. For both scenarios, between 1 and 5 nanoparticles are considered close to the polymersome, distributed across the surface of the vesicle to minimize the interaction between nanoparticles. We have found that the relative orientation of several particles that are either decorated or taken up has a small impact on the overall scattering profile (data not shown).

Cryogenic Transmission Electron Microscopy (CryoTEM). A JEOL1400 transmission electron microscope operated at a $120 \mathrm{kV}$ acceleration voltage was used for recording the cryo-TEM images of the specimens. For cryofixation $^{66}$ of the samples, $5 \mu \mathrm{L}$ of the aqueous solution was dropped onto a Lacey or QUANTIFOIL grid (Lacey support films, NetMesh Grids, Cu-mesh 400; QUANTIFOIL R2/2, Cu-mesh 300). With a Vitrobot (Mark II, FEI), the excess amount of liquid was blotted off, and the sample was frozen in liquid ethane at $T=-178{ }^{\circ} \mathrm{C}$ and transferred to the TEM instrument.

UV-Vis Spectroscopy. All UV-vis measurements were recorded on a PerkinElmer Lambda $25 \mathrm{UV}$-vis spectrometer, using water as a reference.

\section{RESULTS}

System and Advantages. The synthesis of the amphiphilic PDMS- $b$-PMOXA and the fabrication of low polydispersity spherical polymersomes are described elsewhere. $^{44-46,67}$ The utilization of AuNPs has the advantage of localized surface plasmon resonance (LSPR) enhancement of depolarized light scattering even for spherical symmetry ${ }^{65}$ that enables selective detection of particles and vesicles, concurrently at subnanomolar NP concentrations. The vesicles are detectable only in the polarized (VV) light scattering due to their almost spherical shape. The AuNPs are detectable in both $\mathrm{VV}$ and depolarized ( $\mathrm{VH}$ ) light scattering due to the inherent crystallinity of the Au core and the slightly anisotropic surface graft arrangements. ${ }^{65}$ Therefore, information on both translational and rotational AuNP dynamics in the interacting system becomes directly accessible. Engineering the surface chemistry of the AuNPs either by cetyltrimethylammonium bromide 

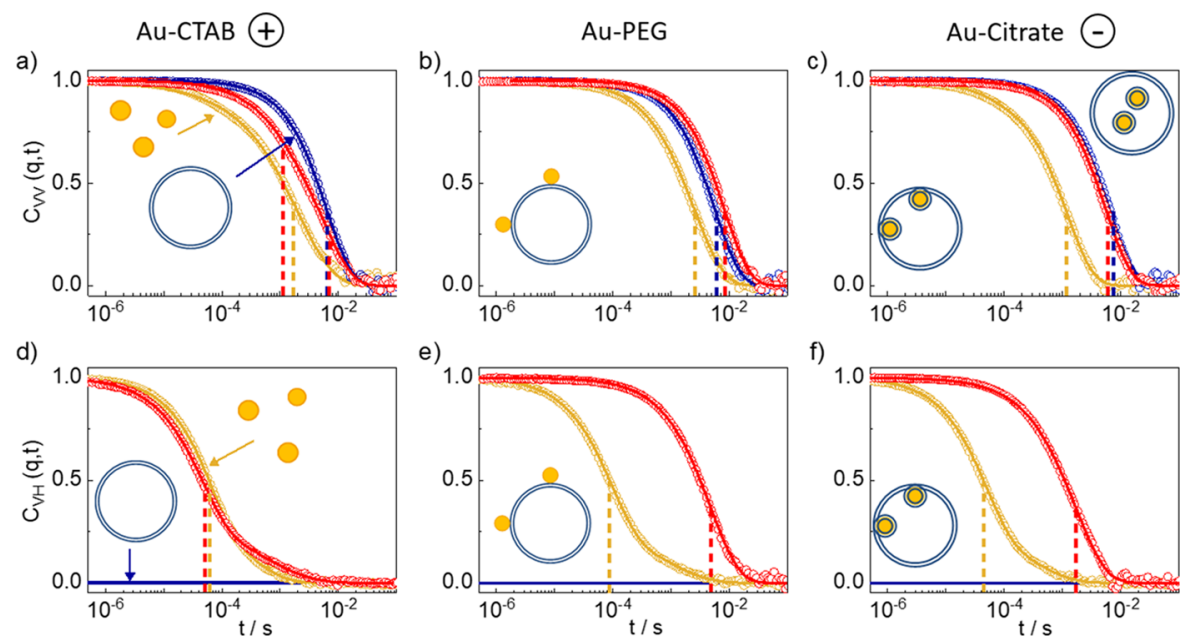

Figure 2. Relaxation functions for the diffusion and rotation dynamics in different vesicle/Au nanoparticle systems. Relaxation functions recorded for $(\mathrm{a}-\mathrm{c}) \mathrm{VV}$ and $(\mathrm{d}-\mathrm{f}) \mathrm{VH}$ polarization at a scattering wave vector $q=1.01 \times 10^{-2} \mathrm{~nm}^{-1}$ for dilute aqueous solutions of PDMS- $b$-PMOXA vesicles with three different AuNPs. (a, d) Au-CTAB, (b, e) Au-PEG ${ }_{107}$, and (c, f) Au-citrate. The solid lines indicate the representation by a single stretched exponential (see text) of the experimental relaxation of the vesicle/AuNP mixture (red), and the solutions of the individual constituents, vesicles (blue) and AuNPs (orange). The vertical dashed lines in a-f indicate the values of characteristic relaxation times. (a, d) Au-CTAB, (b, e) $\mathrm{Au}-\mathrm{PEG}_{107}$, and (c, f) Au-citrate exemplify three different vesicle/AuNP interactions indicated by the schemes in the six panels.

(CTAB) or grafting poly(ethylene glycol) (PEG)) or coating (citrate) enables tuning of the interactions with the vesicles.

The characteristic dimensions (hydrodynamic radius, $R_{\mathrm{h}}$ ), the $\zeta$ potential of the polymersomes, and the different AuNPs are listed in Table 1. For the mesoscopic size of the vesicles, VV-DLS was employed at a $5.5 \mu \mathrm{M}$ polymer concentration to record the form factor, yielding the shape, the radius (Figure S1a), and the relaxation functions, leading to the translational diffusion $D^{\mathrm{t}}$ (Figure S1b) and hence the hydrodynamic size, $R_{\mathrm{h}}$. The ultradilute solutions $(0.2 \mathrm{nM})$ of the three AuNPs exhibit both VV- and VH-DLS, leading to the estimation of $R_{\mathrm{h}}$ $=\left(k_{\mathrm{B}} T /\left(6 \pi \eta_{\mathrm{s}} D^{\mathrm{t}}\right)\right)$ and rotational, $R_{\mathrm{r}}=\left[k_{\mathrm{B}} T /\left(8 \pi \eta_{\mathrm{s}} D^{\mathrm{r}}\right)\right]^{1 / 3}$, radii from the experimental $D^{\mathrm{t}}$ and rotational $D^{\mathrm{r}}$ diffusion coefficients and the water viscosity $\eta_{s}$. For all AuNPs, the $\mathrm{VV}$ and $\mathrm{VH}$ scattering intensity patterns are virtually $q$ independent due to their small size $(q R \ll 1)$. The large disparity $R_{\mathrm{r}}>R_{\mathrm{h}}$, which is not compatible with spherical symmetry, can be ascribed to an anisotropic graft distribution that renders the aspect ratio of an otherwise spherical AuNP shape larger than one. ${ }^{65}$ The AuNPs display a single LSPR mode at $\lambda_{\max }$ which conforms to a spherical Au core with anisotropic graft distribution. ${ }^{65}$ The induced nonspherical shape rationalizes the ligand-dependent intensity depolarization ratio, $R_{\mathrm{VH}} / R_{\mathrm{VV}}$, (Table 1 ), whereas its relatively large value is partly due to the Au core crystallinity.

Realization of Interaction Patterns. To distinguish between the different interaction scenarios of Figure 1, we kept the same vesicle system and utilized the same Au core ( $d$ $=44 \mathrm{~nm}$ ) but bearing different surface charges. The selected coatings allow charge tuning from positive to negative as indicated by the $\zeta$ potential in Table 1 . Both vesicles and AuNPs were thoroughly characterized prior to their mixing at a AuNP/vesicle molar ratio of 5:1. For the vesicle/Au-CTAB solution, the $\mathrm{VV}$ light scattering intensity pattern, $R_{\mathrm{VV}}(q)$, is represented by the sum of the $q$-dependent $R_{\mathrm{VV}}(q, \mathrm{ves})$ of the vesicle and the $q$-independent $R_{\mathrm{VV}}(\mathrm{Au}-\mathrm{CTAB})$ individual solutions (Figure S2a). This result clearly indicates the absence of discernible interactions between the two positively charged species, which is more sensitively reflected in the depolarized
$R_{\mathrm{VH}}$ intensity of the mixture. Due to the entirely isotropic scattering of the vesicles $\left(R_{\mathrm{VH}}(\mathrm{ves}) \approx 0\right), R_{\mathrm{VH}}$ of the mixture should arise from either free AuNPs and/or ves/AuNP complexes. For the vesicle/Au-CTAB, however, $R_{\mathrm{VH}}$ is due entirely to the $R_{\mathrm{VH}}(\mathrm{Au}-\mathrm{CTAB})$ (Figure S2a). It can therefore be concluded that the size, shape, and structure of the vesicles are robust in the vesicle/Au-CTAB system.

A strong confirmation of the noninteracting species stems from the relaxation functions $C_{\mathrm{VV}}(q, t)$ and $C_{\mathrm{VH}}(q, t)$ in the mixture and its constituent components in Figure 2a,d. The relaxation functions $C_{\mathrm{VH}}(q, t)$ for the mixture and $\mathrm{Au}-\mathrm{CTAB}$ $\mathrm{NP}$ solution are identical (Figure $2 \mathrm{~d}$ red and orange curves, see also Figure S2b), yielding the rotational diffusion $D^{\mathrm{r}}$ (and hence $R_{\mathrm{r}}$ ) of the AuNP; for the isotropic vesicle suspension, $C_{\mathrm{VH}}(q, t)=0$ (blue baseline in Figure $2 \mathrm{~d}$ ). The functions $C_{\mathrm{VV}}(q, t)$ are less selective due to contributions of all species in the mixture, but their analysis at different wave vectors renders their resolution unique. For the mixture, $C_{\mathrm{VV}}(q, t)$ (Figure 2a, red) is represented by the sum of the relaxation functions of the mixture components (orange and red lines in Figure 2a). Due to the decrease of the intensity ratio, $R_{\mathrm{VV}}(q, \mathrm{ves}) / R_{\mathrm{VV}}(\mathrm{Au}-$ $\mathrm{CTAB}$ ), with increasing $q$ (Figure $\mathrm{S} 2 \mathrm{a}), C_{\mathrm{Vv}}(q, t)$ resembles more either the vesicle (at low $q$ values) or Au-CTAB relaxation functions. The coexistence and hence the lack of interactions between the vesicles and the Au-CTAB NPs are clearly demonstrated and are conceivable in view of the electrostatic repulsions of positively charged particles (Table $1)$.

The same experiments were conducted for vesicles and $\mathrm{Au}$ PEG NPs bearing an essentially neutral surface (Table 1). The relaxation functions $C_{\mathrm{VV}}(q, t)$ and $C_{\mathrm{VH}}(q, t)$ of the mixture now reveal different relations to the constituent components (Figure $2 \mathrm{~b}, \mathrm{e}$ ) caused by their interactions. The distinct anisotropic dynamic scattering from the mixture being much slower than for the Au-PEG NPs, as clearly indicated by very different rates $\Gamma_{\mathrm{VH}}(q)$ (red and orange vertical dashed lines in Figure 2d). The rotational radius $R_{\mathrm{r}}=207 \pm 13 \mathrm{~nm}$ obtained from the rotational diffusion, $D^{\mathrm{r}}=\Gamma_{\mathrm{VH}}(q) / 6$, is much larger than the radius $(40 \mathrm{~nm})$ of the Au-PEG NP (Table 1). Since 

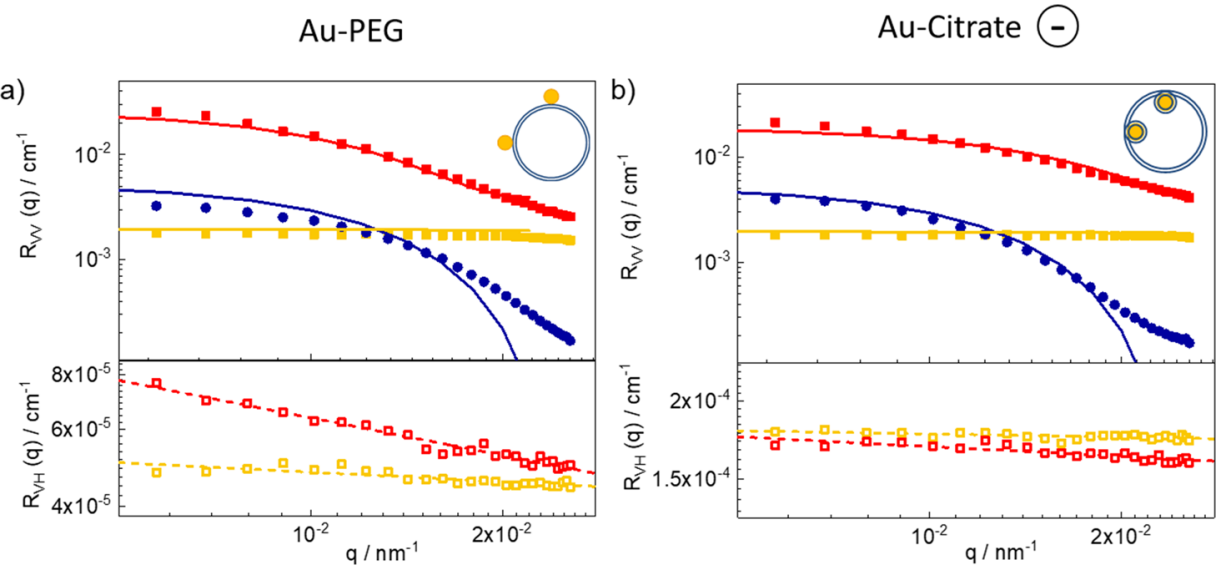

Figure 3. Scattering intensity patterns for interacting vesicle/Au nanoparticle systems. Absolute Rayleigh ratio for polarized $R_{\mathrm{VV}}(q)$ (solid symbols) and depolarized $R_{\mathrm{VH}}(q)$ (open symbols) light scattering from a dilute $\left(c=0.036 \mathrm{~g} \mathrm{~L}^{-1} ; c_{\mathrm{M}}=0.045 \mathrm{nM}\right)$ aqueous suspension of PDMS- $b$-PMOXA vesicles $\left(R_{\mathrm{h}}=135 \pm 3 \mathrm{~nm}\right)$, (a) Au-PEG $(R($ TEM $)=22 \pm 2 \mathrm{~nm})$, and $(\mathrm{b})$ Au-citrate as a function of the scattering wave vector, $q$. The solid lines indicate the theoretical representations (see text) of the experimental intensity patterns of the vesicle/AuNP mixture (red), and the solutions of the individual constituents, vesicles (blue) and AuNP (yellow). (a) Au-PEG 107 and (b) Au-citrate exemplify two different vesicle/AuNP interactions indicated by the schemes in the insets of the two panels. Note that $R_{\mathrm{VH}}(q)$ is higher in b due to larger depolarization ratio of Au-citrate NP (Table $1)$.

the anisotropic VH scattering originates from the AuNPs, the absence of fast dynamics suggests predominantly bound AuNPs, and the large $R_{\mathrm{r}}$ implies assembly of vesicle/Au-PEG NP. A similar but less pronounced trend is observed in the case of $C_{\mathrm{VV}}(q, t)$ that exhibits a slower translation diffusion rate in the mixture than in the vesicle suspension (blue and red dashed vertical lines in Figure 2b). Hence, the vesicle/Au-PEG NP construct with $R_{\mathrm{h}}=190 \mathrm{~nm}$ is clearly larger than the vesicle radius, and moreover, the inequality $R_{\mathrm{r}}>R_{\mathrm{h}}$ suggests deviation from the spherical symmetry. This trend along with the larger size of the vesicle/Au-PEG NP (compared to the bare vesicle) renders a decoration scenario, as illustrated in the insets of Figure $2 \mathrm{~b}$,e, conceivable. This is tested by the intensity $R_{\mathrm{VV}}(q)$ and $R_{\mathrm{VH}}(q)$ (Figure $3 \mathrm{a}$ ) next.

For the vesicle/Au-PEG NP mixture, the intensity $R_{\mathrm{VV}}(q)$ is higher than the sum of the corresponding form factors of the vesicles (blue) and Au-PEG NPs (orange), and $R_{\mathrm{VH}}(q)$ exceeds that of the NPs exhibiting a discernible $q$ dependence, as seen in Figure 3a. Both findings indicate the formation of nonspherical vesicle/Au-PEG NP structure being larger than the spherical vesicles in conformity with the dynamics of Figure $2 \mathrm{~b}$,e. In the decoration scenario, we have computed form factors of different vesicle/NP configurations (Methods Section) and compared (red line) with the experimental $R_{\mathrm{VV}}(q)$. The models were scaled to reproduce the experimental scattering intensity profiles for the vesicle and Au-PEG NPs alone. Discrepancy at a small length scale (near the minimum of the form factor of a sphere) for the vesicle (blue curves in Figure 3) is due to modeling of vesicles of a single size. The computed form factor for the illustrated vesicle +2 Au-PEG NP assembly leads to excellent agreement with the experimental data (red solid lines in Figure 3 and Figure S3a). The topology of the AuNPs on the vesicle surface impacts the optical anisotropy of the structure that sensitively determines $R_{\mathrm{VH}}(q)$. A comparison of these curves between the AuNP and the vesicle/AuNP mixture hints at the supramolecular geometry of the assembly. While Au-citrate alone or mixed with vesicles exhibits very similar optical anisotropy curves, significant deviations are observed for Au-PEG NPs in the presence of vesicles (Figure 3a). The depolarized scattering intensity profiles can be analyzed in terms of a simple theoretical model of a supramolecular assembly that only considers different polarizability contributions along each direction (Section S1). For Au-PEG NPs (Figure 3a), we find that the anisotropy $\delta$ of $\sim 0.05$, obtained from $R_{\mathrm{VH}}(q>0)$, is commensurate with a cylindrical geometry, in which the two main axes contribute with a $\beta / \alpha$ ratio of $\sim 0.86$. Geometrically, this ratio of polarizabilities matches the inertia tensor of an arrangement of two AuNPs decorated on the vesicle at a $90^{\circ}$ angle of one another. The proposed arrangement suggests that the driving force behind the decoration of two AuNPs is purely entropic (Section S1), suggesting that the two AuNPs are randomly and evenly placed on a sphere. Further, this independent placement on the NPs ignores possible contribution from the elastic energy of interaction.

The third vesicle/AuNP assembly architecture was realized upon mixing the vesicle suspension with the solution of the negatively charged Au-citrate NPs. According to Figure 3b, $R_{\mathrm{Vv}}(q)$ for the vesicle/Au-citrate mixture (red symbols) is higher than the summed contributions of the two mixture constituents (blue and orange symbols), but the $q$ dependence is weaker than for the vesicle/Au-PEG system (Figure 3a). This finding indicates that the formed structure is smaller in the vesicle/Au-citrate mixture as also reflected in the faster translation and rotation rates (vertical lines) of the relaxation functions in Figure 2c,f compared to the free vesicles. Note that this trend is better seen in the rotation rate (Figure 2f) due to its $R_{r}^{3}$ dependence. The absence of fast rotation times, reminiscent of free AuNPs, corroborates the notion that the $\mathrm{Au}$-citrate NPs cannot rotate freely (Figure 1c) in the interior of the vesicles. To reveal the new structure, modeling of the NP uptake scenario, schematically shown in the insets of Figure $2 c, f$ and Figure $3 b$, was performed. The uptake mechanism assumes a consumption of the vesicle block copolymer by the endocytosed NPs represented by a shell with a thickness of $20 \mathrm{~nm}$ and scattering length $\rho_{\text {ves. }}$. The loss of polymer in the vesicle was considered by decreasing the vesicle radius by $10 \mathrm{~nm}$, but the modeling results are largely insensitive to the exact size decrease. The NPs with the bilayer coat were placed in the interior of the vesicle as 
indicated in Figure 1d. The experimental $R_{\mathrm{VV}}(q)$ is well represented by the computed form factor (black solid line in Figure $3 b$ ) of this structure with an unweighted average between one and two NPs (Figure S3b).

The spherical shape and smaller size of the encapsulating vesicles are also inferred from their radii $R_{\mathrm{h}}=123 \pm 3 \mathrm{~nm}$ and $R_{\mathrm{r}}=128 \pm 4 \mathrm{~nm}$ computed from the rates $\Gamma_{\mathrm{VV}}(q)$ and $\Gamma_{\mathrm{VH}}(q)$ of the relaxation functions of Figure $2 \mathrm{c}, \mathrm{f}$. For comparison, the values of $R_{\mathrm{h}}$ and $R_{\mathrm{r}}$ in the three systems are listed in Table 2 .

Table 2. Characteristic Dimensions and Experimental Wavelength Change of the Surface Plasmon Resonance Absorption Peak for the Mixtures of Vesicles and Au Nanospheres with Different Surface Graftings

\begin{tabular}{c|ccc} 
& $\begin{array}{c}R_{\mathrm{h}} \\
{[\mathrm{nm}]}\end{array}$ & $\begin{array}{c}R_{\mathrm{r}} \\
{[\mathrm{nm}]}\end{array}$ & $\Delta \lambda_{\max }[\mathrm{nm}]$ \\
\hline $21.3 \pm 0.5$ & $33.1 \pm 0.3$ & +1 \\
$139 \pm 8$ & - & +4 \\
$190 \pm 12$ & $207 \pm 13$ & +8 \\
0 & $123 \pm 3$ & $128 \pm 4$ & +8
\end{tabular}

The vesicle/Au-citrate assembly is about $12 \mathrm{~nm}$ smaller than the original vesicles due to the endocytotic uptake membrane consumption upon particle invagination. The amount of consumed membrane material corresponds to about three wrapped AuNPs in agreement with the molar mass ratio of vesicles to AuNP and the modeling of $R_{\mathrm{VV}}(q)$. The uptake of negatively charged (citrate-stabilized) AuNPs into the polymersomes is accompanied by immobilization of the endosomes as illustrated in Figure 1d.

Additional Evidence of the Interaction Patterns. Discrimination between the different scenarios in Figure 1 is based solely on the analysis of the DLS experiment utilizing the advantage of the concurrent detection of vesicles and AuNPs due to their optical anisotropy and the LSPR-enhanced light scattering. However, descriptive images can be obtained by real-space cryo-TEM, recognizing possible structural alterations inherent to the application of this technique. Moreover, UV-vis extinction spectra utilize LSPR as the sensitive probe of subtle changes in the chemical environment at the surface of AuNPs.

To complement the DLS results, cryo-TEM images of the three vesicle/AuNP mixtures were recorded. Figure 4a-I shows that there is no interaction between vesicles and $\mathrm{Au}-\mathrm{CTAB}$ NPs and that both species coexist. Figure 4a-II,III shows decorated vesicles with Au-PEG 107 NPs, but the particles are not engulfed by the membrane. Instead, these particles seem to slightly cave into the membrane. It should be noted that $4 a-$ II,III look similar, but in 4aII, there is no outer membrane surrounding the particles on the right. Figure 4a-IV shows the uptake of Au-citrate NPs into the vesicles, clearly displaying the membrane around the particles. Some of those endosomes seem to be attached to the membrane, while others seem to diffuse freely within the vesicle. However, as it was shown by DLS, only one species is detected in depolarized PCS, and therefore the presence of free AuNP-endosomes is not supported. Since cryo-TEM shows the transmission and hence a two-dimensional picture only, the apparent free gold endosomes must be anyhow attached to the sides of the membrane. Hence, the apparently free AuNP endosome in last a)
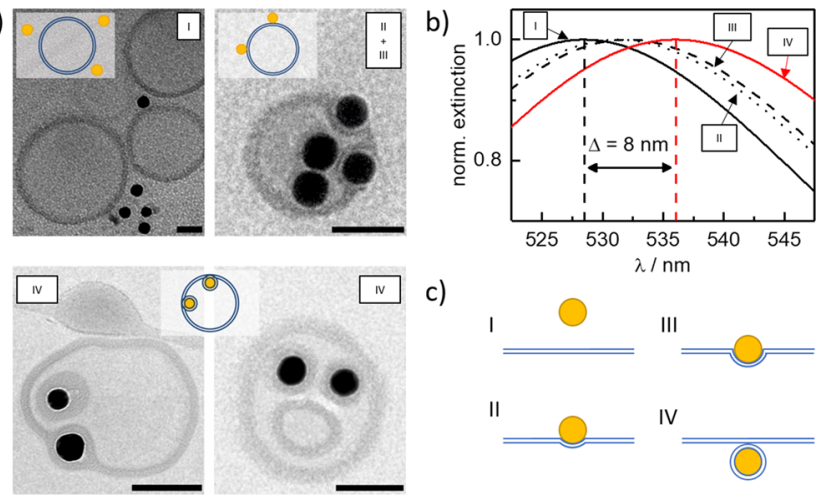

Figure 4. Examination of NP/polymersome interaction patterns. (a) Cryo-TEM images for the three polymersome/AuNP mixtures: (I) $\mathrm{Au}-\mathrm{CTAB},(\mathrm{II}+\mathrm{III}) \mathrm{Au}-\mathrm{PEG}$, and (IV) Au-citrate. The three different polymersome/AuNP interactions are indicated by the schemes in the insets. The last scheme can, in principle, have two realizations (Figure $1 \mathrm{c}, \mathrm{d})$, where the incorporated NP can be attached to or detached from the membrane. The image in the lower right additionally shows a so-called "pregnant" vesicle, which can occur during the film hydration procedure. The scale bar corresponds to $100 \mathrm{~nm}$. (b) Computed UV-vis spectra around the resonance wavelength by the Green's tensor method for a single AuNP, assuming the scenarios in Figure 1: naked AuNP in water (Figure 1a) as shown by the black solid line, only one-third of the particle is covered by the vesicle (Figure $1 \mathrm{~b}$ ) as shown by the black dotted line, one-half of the particle is covered as shown by the black dashed line, and the particle is fully coated with a bilayer (Figure 1d) as shown by the red solid line. To match the $8 \mathrm{~nm}$ wavelength shift found in the experiment, the refractive index of the vesicle was set to 1.45. (c) Schematic illustration of the four scenarios (I-IV).

image of Figure $4 \mathrm{a}$ can be illusive. In a similar context, the apparent size difference by a factor of two in the imaged vesicles in Figure 4a should not be taken as a representative distribution for the whole sample. Cryo-TEM is not a suitable method for determining the absolute size of vesicular structures due to dissimilar compression of the different vesicles between the water interfaces. TEM is a contact and invasive technique in contrast to DLS and in addition not ensemble representative. Hence, TEM cannot unambiguously reveal the different vesicle/NP constructs but support the vesicle/NP interaction patterns inferred by DLS.

The experimental extinction spectra (Figure S4) are compared with calculations using the Green's tensor method for a single AuNP in water, partially covered with a material of refractive index of 1.45 , modeling the vesicle bilayer (Section S2). To describe the four different scenarios pictured on Figure 1 , four cases have been considered in the calculations of the UV-vis spectra in the spectral region around the resonance wavelengths shown in Figure $4 \mathrm{~b}$. These scenarios, AuNP in water (no vesicle, scenario (I)), AuNP half-covered or covered up to one-third of its diameter (corresponding to AuNP touching the external side of the vesicle, scenario (II, III), or AuNP fully wrapped in a shell of bilayer (IV)), are illustrated in Figure 4c. To approximate the resonance wavelengths corresponding to scenario (II, III), the plasmon wavelengths for naked and fully covered AuNPs (Table 2) were compared to those extracted from exact Mie simulations of fully covered AuNP, with a shell of 0,8 , and $16 \mathrm{~nm}$ of the material with a refractive index of 1.45 (Figure $4 \mathrm{~b}$ and Figure S4). Then, the resonance wavelengths for the one-third- and half-covered AuNPs were extracted from a linear regression of those values. 
For the refractive index used to model the vesicle, the wavelength shift is about $8 \mathrm{~nm}(7.5 \mathrm{~nm})$ between naked and fully covered AuNP, while a half- or one-third-covered particle results in a redshift of about $3 \mathrm{~nm}$ (see Table $S$ ). The calculations capture the experimentally observed redshift of the LSPR peaks (black and red vertical dashed lines in Figure $4 b$ ), and accordingly, the up-taken Au-citrate NPs (Figure 2c,f) should be fully wrapped by the membrane, causing the largest $(\sim 8 \mathrm{~nm})$ redshift (Table 2$)$. In contrast, the Au-PEG NPs should be at most half-covered, displaying a lower $(\sim 4 \mathrm{~nm})$ redshift. Hence, a consistent picture of the different interactions has emerged by optimally combining three different experimental techniques and theoretical modeling, and the realization of the proposed patterns is confirmed.

\section{DISCUSSION}

The observed vesicle and AuNP complex depends on the attraction potential, which in turn is determined by the coating of the AuNPs as indicated by their potential in Table 1. For the strongly negatively charged Au-citrate NPs, encapsulation was observed given the positively charged vesicles (Figures 2-4). For the barely negative Au-PEG 107 NPs, the vesicles were decorated with NPs, whereas for the Au-CTAB NPs, the strongly repulsive interactions precluded contacts with the vesicles. Hence, three out of the four possible scenarios depicted in Figure 1 have been realized. We rationalize the three types of interactions by balancing vesicle bending and electrostatic/van der Waals Au NP and vesicle adhesion. The minimal adhesion energy required for wrapping is $8 \pi \kappa /$ $\left(4 \pi R^{2}\right),{ }^{15,68,69}$ where the bending rigidity $\kappa=1.75 \times 10^{-18} \mathrm{~J}$ and the radius of the polymer membrane coating the AuNP (radius, $22 \mathrm{~nm}$ ) is $R=30 \mathrm{~nm}$; the value of $\kappa$ is by a factor of four lower than reported ${ }^{67}$ due to the assumption of a bilayer. Hence, the minimal adhesion energy approximately amounts to about $1 k_{\mathrm{B}} T \mathrm{~nm}^{-2}$. An estimation of the adhesion energy between oppositely charged surfaces is not that straightforward as it depends very strongly on detailed assumptions at a very local range. Envisioning this energy as the difference for two planar surfaces in contact and at large separation, the entropic gain of the free ions alone is too low. Instead, considering the NP uptake like neutralization between an acid and a base, the production of water can release much energy. This occurs when two electric double layers compensated by $\mathrm{H}_{3} \mathrm{O}^{+}$and $\mathrm{OH}^{-}$are brought together.

To rationalize the events (Figures 1 and 4), we utilize the Derjaguin, Landau, Verwey, and Overbeek (DLVO) potential energy for two spheres, one being the vesicle $\left(R_{1}=135 \mathrm{~nm}\right.$; surface potential, $\left.y_{1}=+28 \mathrm{mV}\right)$. The other sphere is one of the $\operatorname{Au}-\mathrm{CTAB}\left(R_{2}=23 \mathrm{~nm} ; y_{2}=+54 \mathrm{mV}\right)$, Au-PEG $\left(R_{2}=37 \mathrm{~nm}\right.$; $\left.y_{2}=-2 \mathrm{mV}\right)$, and Au-citrate $\left(R_{2}=24 \mathrm{~nm} ; y_{2}=-29 \mathrm{mV}\right)$ particles. For the electrostatic double-layer repulsion, we used the value of the Debye length $l_{\mathrm{D}}(300 \mathrm{~nm})$ in distilled water for the van der Waals attraction for solid spheres and the Hamaker constant, $A_{\mathrm{H}}=6 \times 10^{-21} \mathrm{~J}$. For two spheres with surface potentials $y_{1}$ and $y_{2}$ and radii $R_{1}$ and $R_{2}$, the free energy can be calculated as a function of the distance between the surfaces of the two spheres (Section S3). The interaction of Au-CTAB with the polymersome is dominated by electrostatic repulsion between the positively charged side groups, trimethylammonium and oxazoline, respectively (Figure S6). The interaction is repulsive and much larger than $k_{\mathrm{B}} T$ so that coalescence of the particles is prohibited. On the contrary, the interaction between Au-citrate and polymersome is strongly attractive, enabling the observed NP uptake. For the Au-PEG particles, there is a weak attraction to the vesicles allowing for their sticking on the surface of the latter. At low distance, the few 10 $k_{\mathrm{B}} T$ is an indication for a relatively stable adhesion. However, due to the huge difficulties of disentangling local effects (such as hydration or salt bridges), we refrain from attempting a quantitative prediction of its magnitude. At any rate, our experimental data show that the citrate AuNPs with a zeta potential equal to approximately $-k_{\mathrm{B}} T / e$ get wrapped by polymersomes with a zeta potential of approximately $+k_{\mathrm{B}} T / e$, with no other changes in the system, which indicates that electrostatics is the driving force.

The experiment also shows that the driving strength for wrapping is limited because we do not see polymersomes filled with many AuNPs. The likely reason for this is that the process of wrapping puts the membrane of an initially tensionless polymersome under increasing tension because the area is reduced, by approximately $4 \pi(30 \mathrm{~nm})^{2} \approx 11,000 \mathrm{~nm}^{2}$ per AuNP, with no concomitant reduction in volume. There also exists a slightly weaker secondary effect, namely, that the inner leaflet of a fully wrapped AuNP has a smaller area than the outer leaflet, which creates a differential strain in the remaining vesicle membrane. If the thickness of a single leaflet is $8 \mathrm{~nm}$, and the wrapping radius is $30 \mathrm{~nm}$; then, this area difference is $4 \pi\left[(34 \mathrm{~nm})^{2}-(26 \mathrm{~nm})^{2}\right] \approx 6000 \mathrm{~nm}^{2}$ per AuNP, about half as big as the overall removed area. This induces differential stresses in the polymersome, which create a spontaneous curvature that opposes further inward budding. Unlike the adhesion/bending balance, which plays out in the same way for every new AuNP, these tensile stresses accumulate with each ingested particle and make it increasingly harder for new ones to enter.

\section{CONCLUSIONS}

From the four possible interaction scenarios between the positively charged vesicles and NPs in Figure 1, three have been realized. While coalescence of the positively charged Au$\mathrm{CTAB}^{+}$was prohibited, decoration with the barely negative $\mathrm{Au}-\mathrm{PEG}$ and encapsulation of $\mathrm{Au}$-citrate ${ }^{-}$being attached to the inner side of the vesicle were experimentally revealed by surface resonance-enhanced dynamic light scattering, surface plasmon resonance, and electron microscopy. We considered vesicle bending and electrostatic/van der Waals Au NP and vesicle adhesion to rationalize the three types of interaction. While the energy required for wrapping of a AuNP can be approximated by a reasonable value of bending rigidity, the adhesion energy between oppositely charged surfaces was estimated considering the DLVO potential energy for two homogeneous spheres, one being the vesicle and the other one of the three Au NPs. Based on these simulations, the adhesion energy that balances vesicle bending is dominated by electrostatic interactions. In spite of the strong adhesion, Aucitrate polymersomes are filled only with few AuNPs. A likely explanation is that the NP wrapping induces tension in the initially tensionless polymersome because the area is reduced per wrapped AuNP. With each ingested particle the accumulated stresses render additional NP uptake increasingly harder. Elucidation of the physical conditions for passive nanoparticle translocation across membranes can be relevant in the rational design of drugs in the absence of specificity in cellular uptake process. 


\section{ASSOCIATED CONTENT}

\section{(3) Supporting Information}

The Supporting Information is available free of charge at https://pubs.acs.org/doi/10.1021/acs.jpcb.9b10469.

Additional dynamic and static light scattering plots, calculated form factor representations, description of anisotropy analysis from polarized data, description of UV-vis calculations, and description of estimation of adhesion energy (PDF)

\section{AUTHOR INFORMATION}

\section{Corresponding Author}

George Fytas - Max Planck Institute for Polymer Research, 55128 Mainz, Germany; IESL-FORTH, 71110 Heraklion, Greece; Email: fytas@mpip-mainz.mpg.de

\section{Authors}

Amelie H.R. Koch - Max Planck Institute for Polymer Research, 55128 Mainz, Germany

Svenja Morsbach - Max Planck Institute for Polymer Research, 55128 Mainz, Germany; 이이.org/0000-0001-9662-8190

Tristan Bereau - Max Planck Institute for Polymer Research, 55128 Mainz, Germany; 이이.orid/0000-0001-9945-1271

Gaëtan Lévêque - Institut d'Electronique, de Micrólectronique et de Nanotechnologie (IEMN), UMR-CNRS 8520, Faculté de Sciences et Technologies, Universite de Lille, 59655 Villeneuve d'Ascq, France; 10 orcid.org/0000-0003-1626-8207

Hans-Jürgen Butt - Max Planck Institute for Polymer Research, 55128 Mainz, Germany; 이이.org/0000-0001-5391-2618

Markus Deserno - Department of Physics, Carnegie Mellon University, Pittsburgh, Pennsylvania 15213, United States

Katharina Landfester - Max Planck Institute for Polymer Research, 55128 Mainz, Germany; ㅇo이.org/0000-00019591-4638

Complete contact information is available at: https://pubs.acs.org/10.1021/acs.jpcb.9b10469

\section{Notes}

The authors declare no competing financial interest.

\section{ACKNOWLEDGMENTS}

G.F. acknowledges the financial support by ERC AdG SmartPhon no. 694977. M.D. acknowledges funding from the NSF under grant CHE 1764257. We thank M. Bernhardt for performing the cryo-TEM imaging.

\section{REFERENCES}

(1) Oberdörster, G.; Oberdörster, E.; Oberdörster, J. Nanotoxicology: An Emerging Discipline Evolving from Studies of Ultrafine Particles. Environ. Health Perspect. 2005, 113, 823-839.

(2) Wang, A. Z.; Langer, R.; Farokhzad, O. C. Nanoparticle Delivery of Cancer Drugs. Annu. Rev. Med. 2012, 63, 185-198.

(3) Li, W.; Chen, X. Gold nanoparticles for photoacoustic imaging. Nanomedicine 2015, 10, 299-320.

(4) Shin, T.-H.; Choi, Y.; Kim, S.; Cheon, J. Recent advances in magnetic nanoparticle-based multi-modal imaging. Chem. Soc. Rev. 2015, 44, 4501-4516.

(5) Oberdörster, G.; Maynard, A.; Donaldson, K.; Castranova, V.; Fitzpatrick, J.; Ausman, K.; Carter, J.; Karn, B.; Kreyling, W.; Lai, D.; Olin, S.; Monteiro-Riviere, N.; Warheit, D.; Yang, H.; ILSI Research Foundation/Risk Science Institute Nanomaterial Toxicity Screening Working Group. Principles for characterizing the potential human health effects from exposure to nanomaterials: elements of a screening strategy. Particle and Fibre Toxicology 2005, 2, 8.

(6) Lewinski, N.; Colvin, V.; Drezek, R. Cytotoxicity of Nanoparticles. Small 2008, 4, 26-49.

(7) Jiang, W.; Kim, B. Y. S.; Rutka, J. T.; Chan, W. C. W. Nanoparticle-mediated cellular response is size-dependent. Nat. Nanotechnol. 2008, 3, 145.

(8) Rascol, E.; Devoisselle, J.-M.; Chopineau, J. The relevance of membrane models to understand nanoparticles-cell membrane interactions. Nanoscale 2016, 8, 4780-4798.

(9) Karp, G. Cell and molecular biology: concepts and experiments; John Wiley \& Sons, 2009.

(10) Doherty, G. J.; McMahon, H. T. Mechanisms of Endocytosis. Annu. Rev. Biochem. 2009, 78, 857-902.

(11) Mailänder, V.; Landfester, K. Interaction of Nanoparticles with Cells. Biomacromolecules 2009, 10, 2379-2400.

(12) Verma, A.; Stellacci, F. Effect of Surface Properties on Nanoparticle-Cell Interactions. Small 2010, 6, 12-21.

(13) Iversen, T.-G.; Skotland, T.; Sandvig, K. Endocytosis and intracellular transport of nanoparticles: Present knowledge and need for future studies. Nano Today 2011, 6, 176-185.

(14) Deserno, M.; Gelbart, W. M. Adhesion and Wrapping in Colloid-Vesicle Complexes. J. Am. J. Phys. Chem. B 2002, 106, 55435552.

(15) Deserno, M.; Bickel, T. Wrapping of a spherical colloid by a fluid membrane. Europhys. Lett. 2003, 62, 767.

(16) Deserno, M. Elastic deformation of a fluid membrane upon colloid binding. Physical Review E 2004, 69, No. 031903.

(17) Tzlil, S.; Deserno, M.; Gelbart, W. M.; Ben-Shaul, A. A Statistical-Thermodynamic Model of Viral Budding. Biophys. J. 2004, 86, 2037-2048.

(18) Zhang, S.; Li, J.; Lykotrafitis, G.; Bao, G.; Suresh, S. SizeDependent Endocytosis of Nanoparticles. Adv. Mater. 2009, 21, 419424

(19) Yuan, H.; Li, J.; Bao, G.; Zhang, S. Variable Nanoparticle-Cell Adhesion Strength Regulates Cellular Uptake. Phys. Rev. Lett. 2010, 105, 138101.

(20) Yi, X.; Shi, X.; Gao, H. Cellular Uptake of Elastic Nanoparticles. Phys. Rev. Lett. 2011, 107, No. 098101.

(21) Dasgupta, S.; Auth, T.; Gompper, G. Shape and Orientation Matter for the Cellular Uptake of Nonspherical Particles. Nano Lett. 2014, 14, 687-693.

(22) Bahrami, A. H.; Raatz, M.; Agudo-Canalejo, J.; Michel, R.; Curtis, E. M.; Hall, C. K.; Gradzielski, M.; Lipowsky, R.; Weikl, T. R. Wrapping of nanoparticles by membranes. Adv. Colloid Interface Sci. 2014, 208, 214-224.

(23) Raatz, M.; Lipowsky, R.; Weikl, T. R. Cooperative wrapping of nanoparticles by membrane tubes. Soft Matter 2014, 10, 3570-3577.

(24) Smith, K. A.; Jasnow, D.; Balazs, A. C. Designing synthetic vesicles that engulf nanoscopic particles. J. Chem. Phys. 2007, 127, No. 084703.

(25) Li, Y.; Chen, X.; Gu, N. Computational Investigation of Interaction between Nanoparticles and Membranes: Hydrophobic/ Hydrophilic Effect. J. Phys. Chem. B 2008, 112, 16647-16653.

(26) Shi, X.; von dem Bussche, A.; Hurt, R. H.; Kane, A. B.; Gao, H. Cell entry of one-dimensional nanomaterials occurs by tip recognition and rotation. Nat. Nanotechnol. 2011, 6, 714.

(27) Vácha, R.; Martinez-Veracoechea, F. J.; Frenkel, D. ReceptorMediated Endocytosis of Nanoparticles of Various Shapes. Nano Lett. 2011, 11, 5391-5395.

(28) Ruiz-Herrero, T.; Velasco, E.; Hagan, M. F. Mechanisms of Budding of Nanoscale Particles through Lipid Bilayers. J. Phys. Chem. B 2012, 116, 9595-9603.

(29) Huang, C.; Zhang, Y.; Yuan, H.; Gao, H.; Zhang, S. Role of Nanoparticle Geometry in Endocytosis: Laying Down to Stand Up. Nano Lett. 2013, 13, 4546-4550.

(30) Yue, T.; Wang, X.; Huang, F.; Zhang, X. An unusual pathway for the membrane wrapping of rodlike nanoparticles and the 
orientation- and membrane wrapping-dependent nanoparticle interaction. Nanoscale 2013, 5, 9888-9896.

(31) Chen, L.; Xiao, S.; Zhu, H.; Wang, L.; Liang, H. Shapedependent internalization kinetics of nanoparticles by membranes. Soft Matter 2016, 12, 2632-2641.

(32) Markin, V. S.; Kozlov, M. M.; Borovjagin, V. L. On the theory of membrane fusion. The stalk mechanism. Gen. Physiol. Biophys. 1984, 3, 361-377.

(33) Chernomordik, L. V.; Kozlov, M. M. Mechanics of membrane fusion. Nat. Struct. Mol. Biol. 2008, 15, 675.

(34) Kozlovsky, Y.; Kozlov, M. M. Stalk Model of Membrane Fusion: Solution of Energy Crisis. Biophys. J. 2002, 82, 882-895.

(35) François-Martin, C.; Rothman, J. E.; Pincet, F. Low energy cost for optimal speed and control of membrane fusion. Proc. Natl. Acad. Sci. U. S. A. 2017, 114, 1238-1241.

(36) Rothen-Rutishauser, B. M.; Schürch, S.; Haenni, B.; Kapp, N.; Gehr, P. Interaction of Fine Particles and Nanoparticles with Red Blood Cells Visualized with Advanced Microscopic Techniques. Environ. Sci. Technol. 2006, 40, 4353-4359.

(37) Geiser, M.; Rothen-Rutishauser, B.; Kapp, N.; Schürch, S.; Kreyling, W.; Schulz, H.; Semmler, M.; Hof, V. I.; Heyder, J.; Gehr, P. Ultrafine Particles Cross Cellular Membranes by Nonphagocytic Mechanisms in Lungs and in Cultured Cells. Environ. Health Perspect. 2005, 113, 1555-1560.

(38) Banerji, S. K.; Hayes, M. A. Examination of Nonendocytotic Bulk Transport of Nanoparticles Across Phospholipid Membranes. Langmuir 2007, 23, 3305-3313.

(39) Le Bihan, O.; Bonnafous, P.; Marak, L.; Bickel, T.; Trépout, S.; Mornet, S.; De Haas, F.; Talbot, H.; Taveau, J.-C.; Lambert, O. Cryoelectron tomography of nanoparticle transmigration into liposome. J. Struct. Biol. 2009, 168, 419-425.

(40) Koltover, I.; Rädler, J. O.; Safinya, C. R. Membrane Mediated Attraction and Ordered Aggregation of Colloidal Particles Bound to Giant Phospholipid Vesicles. Phys. Rev. Lett. 1999, 82, 1991-1994.

(41) van der Wel, C.; Vahid, A.; Sarić, A.; Idema, T.; Heinrich, D.; Kraft, D. J. Lipid membrane-mediated attraction between curvature inducing objects. Sci. Rep. 2016, 6, 32825.

(42) Kita-Tokarczyk, K.; Grumelard, J.; Haefele, T.; Meier, W. Block copolymer vesicles-using concepts from polymer chemistry to mimic biomembranes. Polymer 2005, 46, 3540-3563.

(43) Landau, L. D.; Lifshitz, E. M. Theory of Elasticity (Course of Theoretical Physics); 3rd ed.; Butterworth-Heinemann, 1986, Vol. 7.

(44) Rideau, E.; Dimova, R.; Schwille, P.; Wurm, F. R.; Landfester, $\mathrm{K}$. Liposomes and polymersomes: a comparative review towards cell mimicking. Chem. Soc. Rev. 2018, 47, 8572-8610.

(45) Jaskiewicz, K.; Larsen, A.; Lieberwirth, I.; Koynov, K.; Meier, W.; Fytas, G.; Kroeger, A.; Landfester, K. Probing Bioinspired Transport of Nanoparticles into Polymersomes. Angew. Chem., Int. Ed. 2012, 51, 4613-4617.

(46) Jaskiewicz, K.; Larsen, A.; Schaeffel, D.; Koynov, K.; Lieberwirth, I.; Fytas, G.; Landfester, K.; Kroeger, A. Incorporation of Nanoparticles into Polymersomes: Size and Concentration Effects. ACS Nano 2012, 6, 7254-7262.

(47) Michel, R.; Kesselman, E.; Plostica, T.; Danino, D.; Gradzielski, M. Internalization of Silica Nanoparticles into Fluid Liposomes: Formation of Interesting Hybrid Colloids. Angew. Chem., Int. Ed. 2014, 53, 12441-12445.

(48) Chen, S.; Wang, Z. L.; Ballato, J.; Foulger, S. H.; Carroll, D. L. Monopod, Bipod, Tripod, and Tetrapod Gold Nanocrystals. J. Am. Chem. Soc. 2003, 125, 16186-16187.

(49) Shankar, S. S.; Bhargava, S.; Sastry, M. Synthesis of Gold Nanospheres and Nanotriangles by the Turkevich Approach. J. Nanosci. Nanotechnol. 2005, 5, 1721-1727.

(50) Yuan, H.; Khoury, C. G.; Hwang, H.; Wilson, C. M.; Grant, G. A.; Vo-Dinh, T. Gold nanostars: surfactant-free synthesis, 3D modelling, and two-photon photoluminescence imaging. Nanotechnology 2012, 23, No. 075102.

(51) Chen, J.; Wiley, B.; Li, Z.-Y.; Campbell, D.; Saeki, F.; Cang, H.; $\mathrm{Au}$, L.; Lee, J.; Li, X.; Xia, Y. Gold Nanocages: Engineering Their
Structure for Biomedical Applications. Adv. Mater. 2005, 17, 22552261.

(52) Payne, E. K.; Shuford, K. L.; Park, S.; Schatz, G. C.; Mirkin, C. A. Multipole Plasmon Resonances in Gold Nanorods. J. Phys. Chem. B 2006, 110, 2150-2154.

(53) Fleischer, M.; Zhang, D.; Braun, K.; Jäger, S.; Ehlich, R.; Häffner, M.; Stanciu, C.; Hörber, J. K. H.; Meixner, A. J.; Kern, D. P. Tailoring gold nanostructures for near-field optical applications. Nanotechnology 2010, 21, No. 065301.

(54) El-Sayed, I. H.; Huang, X.; El-Sayed, M. A. Surface Plasmon Resonance Scattering and Absorption of anti-EGFR Antibody Conjugated Gold Nanoparticles in Cancer Diagnostics: Applications in Oral Cancer. Nano Lett. 2005, 5, 829-834.

(55) Saison-Francioso, O.; Lévêque, G.; Boukherroub, R.; Szunerits, S.; Akjouj, A. Dependence between the Refractive-Index Sensitivity of Metallic Nanoparticles and the Spectral Position of Their Localized Surface Plasmon Band: A Numerical and Analytical Study. J. Phys. Chem. C 2015, 119, 28551-28559.

(56) Liu, C.-J.; Wang, C.-H.; Chen, S.-T.; Chen, H.-H.; Leng, W.-H.; Chien, C.-C.; Wang, C.-L.; Kempson, I. M.; Hwu, Y.; Lai, T.-C.; et al. Enhancement of cell radiation sensitivity by pegylated gold nanoparticles. Phys. Med. Biol. 2010, 55, 931-945.

(57) Chen, X.; Chen, Y.; Yan, M.; Qiu, M. Nanosecond Photothermal Effects in Plasmonic Nanostructures. ACS Nano 2012, 6, 2550-2557.

(58) Rørvig-Lund, A.; Bahadori, A.; Semsey, S.; Bendix, P. M.; Oddershede, L. B. Vesicle Fusion Triggered by Optically Heated Gold Nanoparticles. Nano Lett. 2015, 15, 4183-4188.

(59) Bonnaud, C.; Monnier, C. A.; Demurtas, D.; Jud, C.; Vanhecke, D.; Montet, X.; Hovius, R.; Lattuada, M.; Rothen-Rutishauser, B.; Petri-Fink, A. Insertion of Nanoparticle Clusters into Vesicle Bilayers. ACS Nano 2014, 8, 3451-3460.

(60) Mornet, S.; Lambert, O.; Duguet, E.; Brisson, A. The Formation of Supported Lipid Bilayers on Silica Nanoparticles Revealed by Cryoelectron Microscopy. Nano Lett. 2005, 5, 281-285.

(61) Egli, S.; Nussbaumer, M. G.; Balasubramanian, V.; Chami, M.; Bruns, N.; Palivan, C.; Meier, W. Biocompatible Functionalization of Polymersome Surfaces: A New Approach to Surface Immobilization and Cell Targeting Using Polymersomes. J. Am. Chem. Soc. 2011, 133, $4476-4483$.

(62) Olson, F.; Hunt, C. A.; Szoka, F. C.; Vail, W. J.; Papahadjopoulos, D. Preparation of Liposomes of Defined Size Distribution by Extrusion through Polycarbonate Membranes. Biochim. Biophys. Acta 1979, 557, 9-23.

(63) Ziegler, C.; Eychmüller, A. Seeded Growth Synthesis of Uniform Gold Nanoparticles with Diameters of 15-300 nm. J. Phys. Chem. C 2011, 115, 4502-4506.

(64) Hanauer, M.; Pierrat, S.; Zins, I.; Lotz, A.; Sönnichsen, C. Separation of nanoparticles by gel electrophoresis according to size and shape. Nano Lett. 2007, 7, 2881-2885.

(65) Koch, A. H. R.; Lévêque, G.; Harms, S.; Jaskiewicz, K.; Bernhardt, M.; Henkel, A.; Sönnichsen, C.; Landfester, K.; Fytas, G. Surface Asymmetry of Coated Spherical Nanoparticles. Nano Lett. 2014, 14, 4138-4144.

(66) Iancu, C. V.; Tivol, W. F.; Schooler, J. B.; Dias, D. P.; Henderson, G. P.; Murphy, G. E.; Wright, E. R.; Li, Z.; Yu, Z.; Briegel, A.; Gan, L.; He, Y.; Jensen, G. J. Electron cryotomography sample preparation using the Vitrobot. Nat. Protoc. 2006, 1, 2813-2819.

(67) Jaskiewicz, K.; Makowski, M.; Kappl, M.; Landfester, K.; Kroeger, A. Mechanical Properties of Poly(dimethylsiloxane)-blockpoly(2-methyloxazoline) Polymersomes Probed by Atomic Force Microscopy. Langmuir 2012, 28, 12629-12636.

(68) Lipowsky, R.; Döbereiner, H. G. Vesicles in contact with nanoparticles and colloids. Europhys. Lett. 1998, 43, 219-225.

(69) Dinsmore, A. D.; Wong, D. T.; Nelson, P.; Yodh, A. G. Hard Spheres in Vesicles: Curvature-Induced Forces and Particle-Induced Curvature. Phys. Rev. Lett. 1998, 80, 409-412. 